\title{
Impact of patient positioning on radiotherapy dose distribution: An assessment in parotid tumor
}

\author{
Seema Sharma, Shikha Goyal, Sandeep Muzumdar, Durai Manigandan, Puja Sahai, \\ Ahitagni Biswas, Velliyan Subramani, Subhash Chander
}

Department of Radiation Oncology, All India Institute of Medical Sciences, New Delhi, India

Received September 01, 2015; Revised December 04, 2015; Accepted December 10, 2015; Published Online December 20, 2015

\section{Technical Report}

\begin{abstract}
Purpose: We intended to study the impact of patient positioning on the dose distribution within target volume and organs at risk in patients with parotid malignancies treated with 3D conformal radiotherapy (3D-CRT) with photon wedge pair (WP) or intensity modulated radiotherapy (IMRT). Methods: Three patients with a non-Hodgkin's lymphoma of the right parotid gland were consecutively immobilized using thermoplastic cast in 2 positions: supine with head in neutral position (HN) and with head turned $90^{\circ}$ to the left side (HT). Images for treatment planning purpose were acquired in both positions. For both positions, photon WP plans and 5 field IMRT plans were generated, after contouring clinical target volume (CTV), planning target volume (PTV = CTV + $5 \mathrm{~mm}$ margin) and organs at risk (OAR). All plans were evaluated for target coverage and dose to OARs. Results: Both CTV and PTV were apparently larger in HN compared with HT $(31.76 \pm 8.89$ cc, $30.31 \pm 7.83 \mathrm{cc}$ and $62.49 \pm 19.01 \mathrm{cc}$, $58.89 \pm 15.33 \mathrm{cc}$ ) respectively. The CI value for PTV was slightly better for HT compared to HN position in both the WP and IMRT plans. The homogeneity was comparable in both the head positions in case of WP plan. The mean HI of PTV was increased in case of IMRT plan at HT versus HN position (1.108 vs. 1.097). A change in head position from $\mathrm{HN}$ to $\mathrm{HT}$ with wedge pair plan resulted in a reduction of

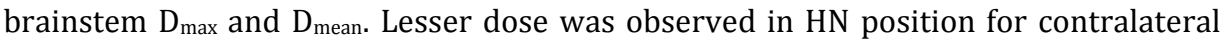
parotid. A difference of $0.9 \mathrm{~Gy}$ in the average $\mathrm{D}_{\max }$ to spinal cord was seen. The values of $\mathrm{D}_{\text {mean }}$ to mandible, oral cavity, ipsilateral and contralateral cochlea were higher in the HT position. A change in head position from HN to HT with IMRT plan resulted in a dose reduction in average $D_{\max }$ to brainstem. The spinal cord $\mathrm{D}_{\max }$ increased at the HT position by $1.2 \mathrm{~Gy}$. The dose to contralateral parotid and cochlea was comparable in both the positions. However, the D mean to oral cavity was reduced at HT position. Whereas for IMRT versus wedge pair plan at head neutral position average $D_{\text {mean }}$ to the contralateral parotid was reduced with the IMRT plan. A considerable reduction in $D_{\max }$ to spinal cord and $\mathrm{D}_{\text {mean }}$ to ipsilateral cochlea was observed. A slight increase in average $D_{\max }$ to brainstem and was observed with the IMRT plan. The doses to the remaining OARs were lesser in case of IMRT plan. For IMRT versus wedge pair plan at head tilt position slight increase in average $D_{\max }$ to brainstem was observed in case of IMRT plan. A considerable reduction in $\mathrm{D}_{\max }$ to spinal cord and $\mathrm{D}_{\text {mean }}$ to ipsilateral cochlea was observed. The doses to the remaining OARs were reduced with IMRT plan. Conclusion: Change in head position from neutral to $90^{\circ}$ contralateral tilt for wedge pair plan in parotid tumor may considerably reduce dose to the brainstem and spinal cord with a modest increase in dose to mandible, oral cavity, contralateral parotid, and bilateral cochlea. The alteration in head position has minimal impact on IMRT planning.
\end{abstract}

Keywords: Conformal Radiotherapy; Dose Distribution; Head Position; Intensity-Modulated Radiotherapy; Parotid Neoplasm 


\section{Introduction}

Newer methods and techniques continue to be explored in radiotherapy practice for achieving the goal of maximum tumor dose with sparing of normal structures. The patient position and immobilization plays a pertinent role in external beam radiotherapy planning. Many authors have studied the influence of treatment position on radiotherapy dose distribution. Wilder et al. ${ }^{1}$ compared the prostate intrafraction motion in prone and supine position author concluded that prone and supine positions resulted in a similar magnitude of anteroposterior (AP) and superoinferior intrafraction prostate motion $(2 \mathrm{~mm})$. Krengli et al. ${ }^{2}$ analyzed the dosimetric parameters of patients receiving adjuvant breast radiotherapy (RT) in prone versus supine position and found that prone position is a favorable alternative for irradiation of mammary gland in patients with pendulous breasts. Sharma et al. ${ }^{3}$ studied the impact of patient position (supine and prone) on patients of brainstem glioma and concluded that supine and prone positions resulted in almost similar dose distribution. Bakkal et $a l^{4}$ evaluated the effect of radiotherapy on testicles with different treatment positions and plans for rectal cancer patients and concluded that supine 4-fields (S4f) external beam radiotherapy for rectal carcinoma allows better testicular dose than prone 3-fields (p3f) and prone 4-fields (p4f). Drzymala et al..$^{5}$ also studied the volume of bowel and dose received in the prone and supine positions in patients undergoing pre-operative rectal cancer chemoradiation, results showed that volume of bowel irradiated is not significantly higher in supine position. The supine with head neutral (HN) treatment position is employed for planning radiotherapy in head and neck cancer patients. A pair of angled wedge photon beam is the most common radiation planning technique for parotid tumors. ${ }^{6,7}$ Since patients with parotid tumors require unilateral irradiation, a change in position from HN to head tilt (HT) may alter the dose distribution. The purpose of this study was to evaluate the influence of head positioning on dose distribution within the target volume and organs at risk (OARs) in patients with parotid tumor. The plans with 3-D conformal radiation therapy (3-DCRT) and intensity modulated radiation therapy (IMRT) were compared alongside the two different head positions.

\section{Methods and Materials}

Three patients with right-sided parotid tumor were treated with radiotherapy. The patients were immobilized using a thermoplastic mould in two positions: supine with head in neutral ( $\mathrm{HN})$ and tilted to the contralateral side at 90 degree (HT). Computed tomography (CT) scan of the head and neck region was performed at $3 \mathrm{~mm}$ slice thickness in both the head positions. The CT images were transferred to the Eclipse $^{\mathrm{TM}}$ (Varian medical system, Palo Alto, CA, Helios version 6.5) treatment planning system (TPS) via
DICOM. The clinical target volume (CTV), planning target volume (PTV), and OARs i.e., contralateral parotid, brainstem, spinal cord, oral cavity, cochlea, and mandible were contoured in both scans for all patients. PTV was generated from CTV with a $5 \mathrm{~mm}$ margin. PTV was intersected with body contour by $2 \mathrm{~mm}$ margin. Two plans with 3-DCRT and IMRT were generated for each of the six CT datasets. The plan with 3-DCRT was generated with a photon wedge pair (WP). Five fields were employed for generating an IMRT plan. The plans were made for Varian CL2300C/D linear accelerator (Varian Medical Systems, Palo Alto, CA, USA) using $6 \mathrm{MV}$ $\mathrm{X}$-rays. The linear accelerator has 40 pairs of multi-leaf collimator (MLC) with a leaf thickness of $1 \mathrm{~cm}$ at isocenter.

\subsection{Dose prescription}

A dose of 60 Gy in 30 fractions was prescribed to the PTV. All plans were prescribed at $95 \%$ isodose line.

\subsection{Wedge pair plan}

The plan was made with two oblique fields using 45 degree wedge. The beam's-eye-view (BEV) option of the TPS was employed to select the appropriate gantry angle to allow coverage of the PTV while minimizing the dose to OARs. The MLC was geometrically shaped around the PTV with a $7 \mathrm{~mm}$ margin for penumbra. A $0.5 \mathrm{~cm}$ bolus was added for achieving a dose build-up at surface.

\subsection{IMRT plan}

The plan was made using five coplanar beams. The gantry angles were $0^{\circ}, 50^{\circ}, 150^{\circ}, 200^{\circ}, 300^{\circ}$ and $0^{\circ}, 72^{\circ}$, $210^{\circ}, 270^{\circ}, 308^{\circ}$ for the respective HN and HT position in case of right-sided parotid tumor. An ipsilateral beam arrangement was employed to provide an optimal dose distribution with maximum sparing of ipsilateral and contralateral OARs. Bolus was not applied in the IMRT optimization. The following dose constraints were imposed for the inverse-planning optimization: atleast $95 \%$ of PTV volume to receive $95 \%$ of prescription dose, brainstem maximum dose $\left(D_{\max }\right) \leq 54 \mathrm{~Gy}$, spinal cord $\mathrm{D}_{\max } \leq 45 \mathrm{~Gy}$, cochlea mean dose (Dmean) $\leq 45 \mathrm{~Gy}$, contralateral parotid gland $D_{\text {mean }} \leq 26 \mathrm{~Gy}$, mandible $\mathrm{D}_{\max }$ $\leq 70 \mathrm{~Gy}$, and oral cavity $\mathrm{D}_{\text {mean }} \leq 45 \mathrm{~Gy}$.

\subsection{Plan evaluation}

All plans were analyzed using DVH. The target coverage was analyzed in terms of minimum, maximum, and mean dose. Coverage index (CI) and heterogeneity index (HI) for PTV were calculated. ${ }^{8}$

$$
\mathrm{CI}=\mathrm{TV}_{\mathrm{RI}} / \mathrm{TV}
$$

where, $\mathrm{TV}_{\mathrm{RI}}=$ target volume covered by the reference isodose; TV =target volume

This index ranges from 0 to 1 . The range of $\mathrm{CI}$ shows the amount of conformation because a value of 1 is rarely achieved. The volume of adjacent healthy tissues is not taken into account in this index. 
$\mathrm{HI}=\mathrm{D}_{5 \%} / \mathrm{D}_{95 \%}$

where, $\mathrm{D}_{5 \%}=$ dose delivered to $5 \%$ of PTV volume; $\mathrm{D}_{95 \%}$ $=$ dose delivered to $95 \%$ of PTV volume

It is evident that if the value of heterogeneity index is closer to 1 , the better will be the dose homogeneity for PTV.

The maximum and mean dose to brain-stem, contra-lateral parotid gland, mandible, oral cavity, bilateral cochlea was noted. Maximum dose to spinal cord was noted.

\section{Results}

The dose distribution for the wedge pair and IMRT plan in neutral and tilted head position is demonstrated in Figure 1.

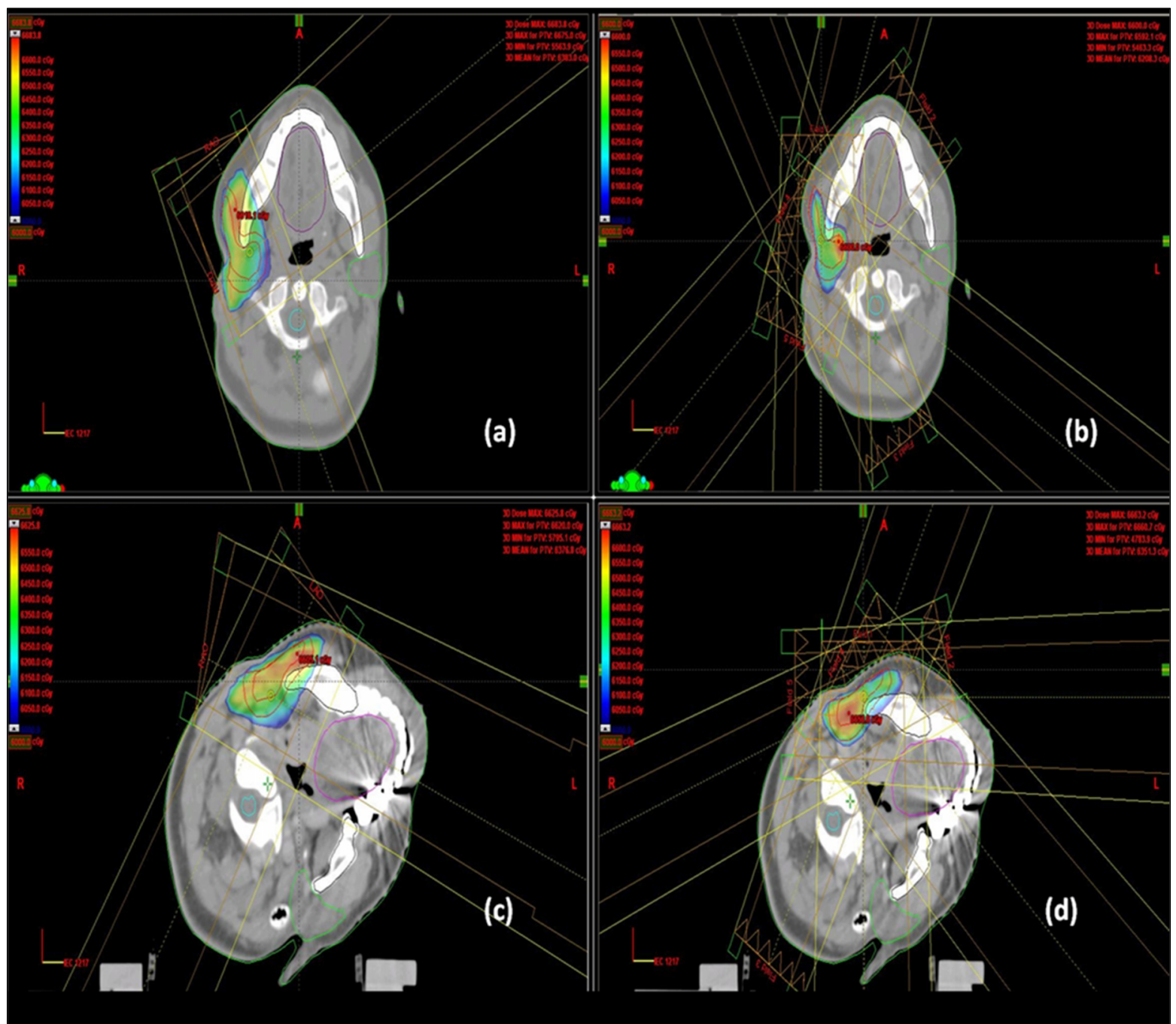

Figure 1: The field setup and isodose line with wedge pair and IMRT plan at neutral (a, b) and tilted (c, d) head position on axial CT slice for a representative patient

Table 1: The target volume for the respective head position.

\begin{tabular}{cc|c|c|c}
\hline \hline & \multicolumn{2}{c}{ Head neutral } & \multicolumn{2}{c}{ Head tilt } \\
\cline { 2 - 5 } & CTV Volume (cc) & PTV volume (cc) & CTV volume (cc) & PTV volume (cc) \\
\cline { 2 - 5 } Patient 1 & 24.30 & 49.50 & 23.80 & 48.50 \\
Patient 2 & 41.60 & 84.30 & 39.00 & 76.50 \\
Patient 3 & 29.38 & 53.66 & 28.12 & 51.68 \\
\hline \hline
\end{tabular}

Abbreviations: CTV = Clinical target volume; PTV = Planning target volume 
Table 2: The target volume dose parameters and indices for the respective head position.

\begin{tabular}{lc:c|c|c}
\hline \hline & \multicolumn{2}{c|}{ Head neutral } & \multicolumn{2}{c}{ Head tilt } \\
\cline { 2 - 5 } PTV Dmax (Gy) & IMRT & WP & IMRT & WP \\
\cline { 2 - 5 } PTV Dmean (Gy) & $66.23 \pm 0.45$ & $67.42 \pm 0.51$ & $66.31 \pm 0.55$ & $67.04 \pm 0.85$ \\
PTV Coverage index (CI) & $63.01 \pm 0.81$ & $64.03 \pm 0.51$ & $63.45 \pm 0.16$ & $63.99 \pm 0.68$ \\
PTV Heterogeneity index (HI) & $0.927 \pm 0.03$ & $0.971 \pm 0.01$ & $0.931 \pm 0.03$ & $0.978 \pm 0.03$ \\
\hline \hline
\end{tabular}

Abbreviations: IMRT = Intensity modulated radiation therapy; WP = Wedge pair; PTV = Planning target volume

Table 3: The maximum and mean doses to organs at risk for the respective head position.

\begin{tabular}{ccc|c|c}
\hline \hline & \multicolumn{2}{c|}{ Head neutral } & \multicolumn{2}{c}{ Head tilt } \\
\cline { 2 - 5 } Brainstem & IMRT & WP & IMRT & WP \\
\cline { 2 - 5 } Dmax (Gy) & $22.99 \pm 7.31$ & $20.71 \pm 11.03$ & $19.81 \pm 5.70$ & $17.38 \pm 8.40$ \\
Dmean (Gy) & $6.83 \pm 4.44$ & $7.29 \pm 2.78$ & $7.67 \pm 3.50$ & $5.40 \pm 4.70$ \\
Contralateral & & & & \\
parotid & & & & \\
Dmax (Gy) & $6.83 \pm 5.65$ & $8.78 \pm 9.49$ & $6.62 \pm 4.37$ & $14.9 \pm 12.14$ \\
Dmean (Gy) & $1.17 \pm 0.49$ & $4.54 \pm 5.52$ & $2.94 \pm 2.65$ & $8.07 \pm 6.50$ \\
Spinal cord & & & & \\
Dmax (Gy) & $19.75 \pm 15.85$ & $26.72 \pm 13.57$ & $20.98 \pm 11.29$ & $25.83 \pm 2.98$ \\
Mandible & & & & \\
Dmax (Gy) & $64.29 \pm 0.31$ & $65.85 \pm 1.21$ & $64.71 \pm 0.27$ & $65.69 \pm 0.42$ \\
Dmean (Gy) & $24.04 \pm 1.76$ & $23.76 \pm 3.21$ & $22.51 \pm 2.97$ & $25.44 \pm 11.01$ \\
Dmax (Gy) & $33.88 \pm 2.92$ & $37.89 \pm 21.69$ & $33.87 \pm 22.81$ & $39.85 \pm 21.69$ \\
Dmean (Gy) & $20.23 \pm 6.74$ & $20.98 \pm 2.87$ & $16.48 \pm 7.29$ & $23.74 \pm 12.19$ \\
Oral cavity & & & & \\
Dmax & $18.57 \pm 11.60$ & $26.25 \pm 7.14$ & $19.28 \pm 10.73$ & $34.71 \pm 12.12$ \\
Dmean (Gy) & $6.06 \pm 3.95$ & $20.96 \pm 9.26$ & $13.31 \pm 6.81$ & $23.59 \pm 12.70$ \\
Ipsilateral cochlea & & & & \\
Contralateral & & & & \\
Dmax (Gy) & $2.65 \pm 1.01$ & $1.97 \pm 0.86$ & $4.56 \pm 3.81$ & $3.50 \pm 2.71$ \\
Dmean (Gy) & $1.57 \pm 0.46$ & $1.60 \pm 0.65$ & $3.74 \pm 3.40$ & $1.94 \pm 0.81$ \\
\hline \hline
\end{tabular}

Abbreviations: IMRT = Intensity modulated radiation therapy; WP = Wedge pair

The volume of CTV and PTV for the HN and HT position is listed in Table 1.

The average volume of CTV and PTV in HN position was $31.76 \pm 8.89 \mathrm{cc}$ and $62.49 \pm 19.01 \mathrm{cc}$, respectively. While the respective values in HT position were $30.31 \pm 7.83 \mathrm{cc}$ and $58.89 \pm 15.33 \mathrm{cc}$. The measured target volume was apparently larger in $\mathrm{HN}$ as compared to the HT position.

The PTV dose parameters and indices are listed in Table 2.

The CI value for PTV was slightly better for HT compared to HN position in both the WP and IMRT plans. The dose distribution in IMRT more closely matched the shape of PTV as compared with the WP plan. Since bolus was not applied for optimization in IMRT, there was under-dosing of PTV in the build-up region. This resulted in lower mean CI value with IMRT in contrast to WP plan.

The homogeneity was comparable in both the head positions in case of WP plan. The mean HI of PTV was increased in case of IMRT plan at HT versus HN position (1.108 vs. 1.097). The best homogeneity was observed with WP plan at HT position (mean HI=1.082).

The doses to OARs with the plans at respective head position are listed in Table 3.

\subsection{Head neutral versus tilt position with wedge pair plan}

A change in head position from HN to HT resulted in a reduction of brainstem $D_{\max }$ and $D_{\text {mean }}$. A major variation in the $D_{\max }$ and $D_{\text {mean }}$ to the contralateral parotid was observed with lesser doses at the HN position (average $\mathrm{D}_{\text {mean }} 4.54$ Gy vs. 8.07 Gy). A difference of 0.9 Gy in the average $D_{\max }$ to spinal cord was seen. The values of $D_{\text {mean }}$ to mandible, oral cavity, ipsilateral and contralateral cochlea were higher in the HT position.

\subsection{Head neutral versus tilt position with IMRT plan}

A change in head position from HN to HT resulted in a dose reduction in average $D_{\max }$ to brainstem (22.99 Gy vs. $19.81 \mathrm{~Gy}$ ). The spinal cord $\mathrm{D}_{\max }$ increased at the HT 
position by $1.2 \mathrm{~Gy}$. The dose to contralateral parotid and cochlea was comparable in both the positions. However, the $\mathrm{D}_{\text {mean }}$ to oral cavity was reduced at HT position.

\subsection{IMRT versus wedge pair plan at head neutral position}

The average $D_{\text {mean }}$ to the contralateral parotid was reduced with the IMRT plan (1.17 Gy vs. 4.54 Gy). A considerable reduction in $\mathrm{D}_{\max }$ to spinal cord and $\mathrm{D}_{\text {mean }}$ to ipsilateral cochlea was observed. A slight increase in average $D_{\max }$ to brainstem and was observed with the IMRT plan (22.99 Gy vs. $20.71 \mathrm{~Gy}$ ). The doses to the remaining OARs were lesser in case of IMRT plan.

\subsection{IMRT versus wedge pair plan at head tilt position}

A slight increase in average $D_{\max }$ to brainstem was observed in case of IMRT plan (19.81 Gy vs. $17.38 \mathrm{~Gy}$ ). A considerable reduction in $\mathrm{D}_{\max }$ to spinal cord and $\mathrm{D}_{\text {mean }}$ to ipsilateral cochlea was observed. The doses to the remaining OARs were reduced with IMRT plan.

\section{Discussion}

Various planning techniques have been described for parotid gland tumour. The ipsilateral WP, WP with lateral portal (3-fields), and the mixed electron-photon beam were considered the optimal techniques in a study by Yaparpalvi et al. ${ }^{9}$ The WP planning has been conventionally described in supine with $\mathrm{HN}$ position. The present study elaborated on dosimetry in tilted head as compared with the neutral position. The target coverage and sparing of normal structures were acceptable with the plans at both head positions. However, an increase in dose to mandible and oral cavity was observed with WP plan at HT position, since the beam entry and exit was unavoidable through these structures in this position. Similarly, IMRT plan in both head positions demonstrated almost same results with respect to the doses to target and OARs.

Nutting et al. ${ }^{10}$ compared the conventional radiotherapy, 3-DCRT, and IMRT for patients with parotid gland tumors. A reduction in the radiation dose to critical normal tissues was demonstrated with 3-DCRT compared with conventional radiotherapy. A further reduction in the dose to the cochlea and oral cavity was observed with IMRT. With nine and seven fields, the dose to the contra-lateral parotid gland was increased, but this was avoided by optimization of the beam directions. The benefits of IMRT were maintained with three or four fields when the beam angles were optimized. Bragg et al. ${ }^{11}$ compared IMRT with 3-DCRT plans for the treatment of tumors of parotid gland. An IMRT class solution of five fields at $15^{\circ}, 55^{\circ}, 125^{\circ}, 165^{0}$, and $270^{\circ}$ was proposed which resulted in improved target dose distribution and sparing of critical structures with an efficient treatment delivery. IMRT reduced the mean dose to the contralateral parotid gland and the maximum doses to the brain and the spinal cord, but increased the ipsilateral lens dose in some cases. A study 12 compared 3D-CRT and IMRT with respect to hearing loss. The mean volume of the inner ear receiving a dose higher than 50 Gy decreased from $14.7 \%$ to $1.4 \%$. Likewise, the current study showed a superior sparing of relatively all OARs with IMRT. A considerable reduction in dose to ipsilateral ear was observed which was not achievable with a WP plan (average Dmean 6.06 Gy vs 20.96 Gy in HN position).

\section{Conclusion}

A change in head position from neutral to $90^{\circ}$ contralateral tilt for wedge pair plan in parotid tumor may considerably reduce dose to the brainstem and spinal cord with a modest increase in dose to mandible, oral cavity, contralateral parotid, and bilateral cochlea. The alteration in head position has minimal impact on IMRT planning. Head position and treatment technique may be selected according to the clinical requirement on an individual case basis.

\section{Conflict of interest}

The authors declare that they have no conflicts of interest. The authors alone are responsible for the content and writing of the paper.

\section{References}

1. Wilder RB, Chittenden L, Mesa AV, et al. A prospective study of intrafraction prostate motion in the prone vs. supine position. Int J Radiat Oncol Biol Phys. 2010;77:165-70.

2. Krengli M, Masini L, Caltavuturo T, et al. Prone versus supine position for adjuvant breast radiotherapy: a prospective study in patients with pendulous breasts. Radiat Oncol. 2013;8:232.

3. Sharma S, Chaudhari P, Biswas A, et al. Impact of head immobilization position on dose distribution in patients of brainstem glioma. Int J Cancer Ther Oncol. 2015; 3:030116.

4. Bakkal BH, Vural T, Elmas 0, et al. Effect of treatment position and radiotherapy planning on testicular dose in patients with rectal carcinoma. J Cancer Res Ther. 2014;10:558-62.

5. Drzymala M, Hawkins MA, Henrys AJ, et al. The effect of treatment position, prone or supine, on dose-volume histograms for pelvic radiotherapy in patients with rectal cancer. $\mathrm{Br}$ J Radiol. 2009;82:321-7.

6. Maes A, Weltens C, Flamen P, et al. Preservation of parotid function with uncomplicated conformal radiotherapy. Radiother Oncol. 2002;63:203-11.

7. Edlund TL. Treatment planning of oblique wedge fields comparing enhanced dynamic wedge and standard 60 degree wedge for 
parotid type treatments. Med Dosim. 1997; 22:197-9.

8. Feuvret L, Noël G, Mazeron JJ, Bey P. Conformity Index: a review. Int J Radiat Oncol Biol Phys. 2006;64:333-42.

9. Yaparpalvi R, Fontenla DP, Tyerech SK, et al. Parotid gland tumors: a comparison of postoperative radiotherapy techniques using three dimensional (3D) dose distributions and dose-volume histograms (DVHS).Int J Radiat Oncol Biol Phys. 1998;40:43-9.

10. Nutting CM, Rowbottom CG, Cosgrove VP, et al. Optimisation of radiotherapy for carcinoma of the parotid gland: a comparison of conventional, three-dimensional conformal, and intensity-modulated techniques. Radiother Oncol. 2001;60:163-72.

11. Bragg CM, Conway J, Robinson MH. The role of intensity-modulated radiotherapy in the treatment of parotid tumors. Int J Radiat Oncol Biol Phys. 2002;52:729-38.

12. Lamers-Kuijper E, Schwarz M, Rasch C, Mijnheer B. Intensity-modulated vs. conformal radiotherapy of parotid gland tumors: potential impact on hearing loss. Med Dosim. 2007;32: 237-45. 